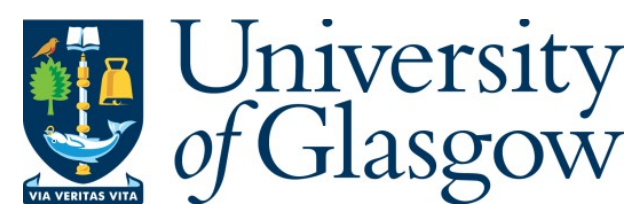

Onireti, O., Imran, A., Imran, M. A., and Tafazolli, R. (2016) Impact of Positioning Error on Achievable Spectral Efficiency in Database-Aided Networks. In: 2016 IEEE International Conference on Communications (ICC), Kuala Lumpur, Malaysia, 22-27 May 2016, ISBN 9781479966646.

There may be differences between this version and the published version. You are advised to consult the publisher's version if you wish to cite from it.

http://eprints.gla.ac.uk/133000/

Deposited on: 19 December 2016

Enlighten - Research publications by members of the University of Glasgow http://eprints.gla.ac.uk 


\title{
Impact of Positioning Error on Achievable Spectral Efficiency in Database-aided Networks
}

\author{
Oluwakayode Onireti, Ali Imran ${ }^{\dagger}$, Muhammad Ali Imran and Rahim Tafazolli \\ Institute for Communication Systems (ICS), University of Surrey, Guildford GU2 7XH, UK \\ ${ }^{\dagger}$ Telecommunications Engineering, University of Oklahoma, Tulsa, OK, USA \\ Email: \{o.s.onireti, m.imran, r.tafazolli\}@surrey.ac.uk and ali.imran@ou.edu ${ }^{\dagger}$
}

\begin{abstract}
Database-aided user association, where users are associated with data base stations (BSs) based on a database which stores their geographical location with signal-to-noise-ratio tagging, will play a vital role in the futuristic cellular architecture with separated control and data planes. However, such approach can lead to inaccurate user-data BS association, as a result of the inaccuracies in the positioning technique, thus leading to suboptimal performance. In this paper, we investigate the impact of database-aided user association approach on the average spectral efficiency (ASE). We model the data plane base stations using its fluid model equivalent and derive the ASE for the channel model with pathloss only and when shadowing is incorporated. Our results show that the ASE in database-aided networks degrades as the accuracy of the user positioning technique decreases. Hence, system specifications for database-aided networks must take account of inaccuracies in positioning techniques.
\end{abstract}

Index Terms - Control and data split architecture, positioning error, database-aided, positioning technique, location error.

\section{INTRODUCTION}

To meet the exponentially growing demand for data rate, researchers in both academia and industry have recently embarked on the design of the fifth generation $(5 \mathrm{G})$ of mobile communications system. In general, three main approaches towards data rate increase have been identified and they are as follows: 1) increasing the area spectral efficiency via network densification and traffic offloading; 2) more bandwidth by moving to the mmWave spectrum and; 3) increasing spectral efficiency via large scale antenna system [1], [2]. The main focus here is on network densification which is achieved via having a heterogeneous network (HetNets), where low-power nodes (small cells) provide high capacity and enhanced user data rates, while the macro cell layer provides ubiquitous coverage.

The conventional approach towards HetNet poses a number of challenges in terms of network management and energy consumption, as a result of the increased number of cells [3]. In order to mitigate these challenges, a new HetNet architecture with split control and data planes has been recently proposed as a candidate architecture for $5 \mathrm{G}$ network [4]-[8]. In such architecture, the control and data planes are separated and are not necessarily handled by the same node. Consequently, this gives the network operator more flexibility, since the small/data cells can be activated on demand to deliver user equipment (UE) specific data only when and where needed, while the macro/control cells manage UEs connectivity and mobility [5], [8]. Hence, the overall energy consumption can be reduced as a result of the on demand activation of the data cells which also leads to longer data cell sleep period.

Several energy saving schemes, such as [9] and [10], have been proposed in literature to put the data cells in a sleep mode when not in use. The action of putting data cells to sleep mode and reactivating them is usually performed via signaling on the data cell radio link. Hence, a fundamental requirement of these schemes is the need to keep the transmitting/receiving part of the radio frequency (RF) circuitry of the data cells on, which potentially leads to an energy consumption overhead [11]. One feasible solution is to switch off the receiving and transmitting RF chains of the data cells. However, if the RF receiving chain of the data cells are switched off, UEs will not be able to connect to the data cells by sending signals on the radio interface, but would require the assistance of the control cell to do so. On the other hand, if the RF transmitting chain of the data cells are switched off, the UEs will not be able to detect the data cells since no pilot signals will be transmitted. In addition, the lack of pilot signal makes it impossible to estimate the current channel between the UEs and the sleeping cells. Hence, putting the data cells in sleep mode deprives the UEs of the information required to achieve optimal data cell association. In order to alleviate this problem the authors in [11], [12] proposed a database-aided energy saving scheme. In the proposed scheme, each control base station (BS) is equipped with a database which stores the geographical channel quality information of all the data cells within its coverage. This enables the data cells to be discovered even when both the transmitting and receiving RF circuitry are turned off.

Accurate association of UEs with the data cells is highly dependent on the accuracy of the positioning technique used in obtaining UEs location. Hence, in this paper, we investigate analytically the impact of inaccuracies in the positioning technique on the average spectral efficiency (ASE) of a typical (random) UE. To the best of our knowledge, this paper presents the first study in this direction. The rest of this paper is organized as follows: In Section II, we present the system model detailing the system architecture, database-aided association procedure, fluid model based data plane model and our approach for modeling the inaccuracies in the positioning technique. In Section III, we derive the ASE for a model based on pathloss only, while Section IV further incorporates 


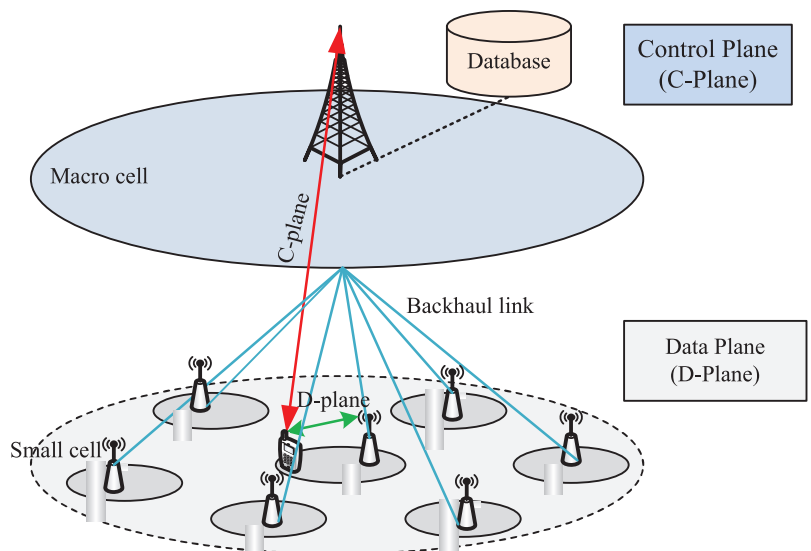

Fig. 1. Control-data plane separated architecture

shadowing in the ASE derivation. In Section V, we present the numerical results. Our results reveal that inaccuracy in the position estimation technique degrades ASE of database-aided networks. Finally, Section VI concludes the paper.

\section{System Model}

\section{A. System Architecture}

In this paper, we consider a cellular architecture with separated control and data planes, as illustrated in Fig. 1. The control plane (C-plane), comprised of control plane BSs or macro cells operating on lower frequency bands (e.g., $2 \mathrm{GHz}$ ), provides control functionalities for all UEs as well as data transmission for low rate and high mobility UEs. Whereas, the data plane (D-plane) comprised of data BSs or small cells operating on higher frequency bands (e.g., $3.5 \mathrm{GHz}$ and beyond) provides high data rate transmission to the UEs. Furthermore, each control plane BS is equipped with a database containing information about the connected data BSs within its coverage.

\section{B. Database-Aided Association}

We consider the scenario proposed in [11], [12] where UEs are enabled to connect with the data BS based on their geographic location information. The control BS is equipped with a database that aids the UE in selecting the most appropriate data BS within the coverage of the control cell. The database is divided into partition, where each partition corresponds to a data BS connected to the control BS. Within each of the partitions, the signal-to-noise-ratios (SNR) of the links between the UEs and the corresponding data BSs are stored and mapped to a set of geographical coordinates $\left(x_{i}, y_{i}\right)$. One major application of such database-aided approach is the possibility of estimating a data cell channel, even when the data cell is in sleep mode and thus reactivating if necessary. However, the geographical coordinates of the UE has to be obtained in order to retrieve the SNR value from the database at the appropriate UE location. UE location information can be obtained by using positioning techniques, such as observed time difference of arrival (OTDOA) or assisted global positioning system
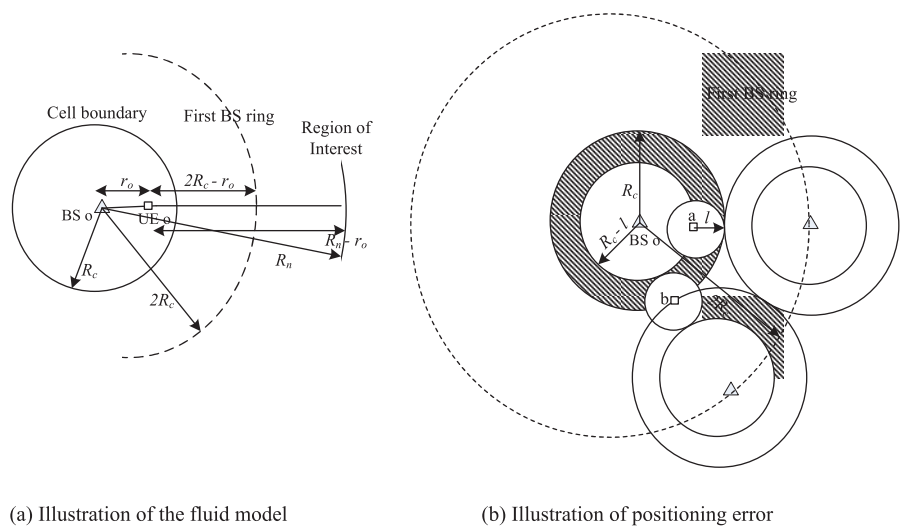

(b) Illustration of positioning error

Fig. 2. Fluid model showing region and cell of interest

(A-GPS) [13]. These techniques are however prone to some errors. For example, the accuracy of A-GPS was evaluated as $10 \mathrm{~m}, 10-20 \mathrm{~m}$ and $10-100 \mathrm{~m}$ for rural, suburban and urban environments, respectively [13]. Consequently, inaccuracies in UE location estimation would result in inaccurate UE-data BS association and, hence, suboptimal performance.

\section{D-plane System Model}

In this paper, we focus on the downlink channel. To keep the analytical tractability, the data plane is modeled to be comprised of homogeneous circular data cells of radius $R_{c}$, in which each data BS having omnidirectional antenna is located at the center and transmit a fixed power $P_{e}$. A frequency reuse 1 is assumed, i.e., all data BSs transmit on the whole system bandwidth. We model the fixed finite number of data BSs by an equivalent density of data BSs according to some distribution function which follow the fluid model [14]. We consider the cell located at the center of the region of interest (ROI) and a UE located inside the cell, as illustrated in Fig. 2(a). We refer to them as the reference cell and reference UE, respectively. The reference cell is surrounded by rings of cells located from $2 R_{c}$ to $R_{n}$.

\section{Modeling Location Estimation Inaccuracy}

We model the location inaccuracy as a result of the position estimation technique using the probability of inaccurate association. Consider the reference UE in the reference data cell, $o$, the uncertainty region as a result of the location inaccuracy can be modeled either one-dimensional or two-dimensional. Here we focus on the 2-dimensional case, since the 1 -dimensional case is more suited to a linear highway cellular architecture. Given an actual location for the reference UE, the reference UE reported location (obtained via a position estimation technique) will lie within a circular area with radius, $l$, centered at the actual location ${ }^{1}$. We consider that the UEs are associated with the closest BS and that $l \leq \frac{R_{c}}{2}$. Hence, in the fluid model illustration of Fig. 2(b), the reference

\footnotetext{
${ }^{1}$ Note that given the reported location, the actual location lies within a circular area with radius $l$, centered at the reported location.
} 
UE is characterized with the probability of association with the reference data $\mathrm{BS}$ and a data $\mathrm{BS}$ in the first ring given by $\mathcal{P}\left(r_{o}, l\right)$ and $\left(1-\mathcal{P}\left(r_{o}, l\right)\right)$, respectively, where $r_{o}$ is the actual distance to the reference data cell. The probability of association with the reference data cell can be expressed from [15] as follows

$$
\mathcal{P}\left(r_{o}, l\right)=\frac{\beta-\sin \beta}{2 \pi}-\frac{\theta-\sin \theta}{2 \pi}\left(\frac{R_{c}}{l}\right)^{2},
$$

for the 2 -dimensional case, where $\beta=2 \cos ^{-1}\left[\frac{r_{o}^{2}+l^{2}-R_{c}^{2}}{2 r_{o} l}\right]$ and $\theta=2 \cos ^{-1}\left[\frac{R_{c}^{2}+r_{o}^{2}-l^{2}}{2 r_{o} R_{c}}\right]$.

\section{Average Spectral Efficiency: Pathloss Only}

\section{A. No error in location information}

Assuming that the distance dependent pathloss is the dominant factor when modeling the signal propagation, the received signal at the reference UE from the reference data BS can be expressed as

$$
P_{r, o}=P_{e} K r_{o}^{-\alpha},
$$

where $r_{o}^{-\alpha}$ is the standard power-law pathloss with exponent $\alpha$ and $K$ is the pathloss at reference distance. We now drop the index $o$ and set $r_{o}=r$. The signal-to-interference-plusnoise-ratio (SINR) experienced by the reference UE can be expressed as

$$
\gamma=\frac{P_{e} K r^{-\alpha}}{\sum_{j \neq o} P_{e} K r_{j}^{-\alpha}+N},
$$

where $r_{j}$ is the distance between data BS $j$ and the reference UE, $N$ denotes the noise power. For an interference limited network, the SINR can be approximated as the signal-tointerference-ratio (SIR) which is given according to the fluid model [14] as

$$
\gamma \approx \widetilde{\gamma}(r)=\frac{r^{-\alpha}}{\sum_{j \neq o} r_{j}^{-\alpha}} \approx \frac{r^{-\alpha}(\alpha-2)}{2 \pi \lambda\left(2 R_{c}-r\right)^{2-\alpha}}
$$

Consequently, the ASE over the reference cell is expressed as

$$
\mathcal{C}_{n}=\int_{d_{0}}^{R_{c}} \ln (1+\widetilde{\gamma}(r)) p_{r}(r) \mathrm{d} r,
$$

where $p_{r}(r)=\frac{2\left(r-d_{0}\right)}{\left(R_{c}-d_{0}\right)^{2}}, \quad d_{0} \leq r \leq R_{c}$, is the probability density function (PDF) of the UE position relative to its serving data BS [16]. $d_{0}$ corresponds to the closest distance the UE can be from the BS antenna.

\section{B. Error in location information}

The case with error in UE location estimation is depicted in Fig. 2b. It can be seen that irrespective of the location error, the reference UE will be connected to the right data BS (i.e. the reference data $\mathrm{BS}$ ) as long as it lies within the range $R_{c}-l$ from the reference data $\mathrm{BS}$, (since $\mathcal{P}(r, l) \triangleq 1$ in this case). However, for the reference UE with actual position that falls in the range $R_{c}-l$ to $R_{c}$, it will be associated with the reference data BS with probability $0<\mathcal{P}(r, l)<1$ and with a data $\mathrm{BS}$ in the first ring with probability $1-\mathcal{P}(r, l)$. The received signal by the UE as a result of association to a data BS in the first ring is given by

$$
\bar{P}_{r}=P_{e} K\left(2 R_{c}-r\right)^{-\alpha}
$$

while the total interference experienced due to such association is expressed as

$$
\begin{aligned}
\bar{I}_{e x t} & \stackrel{(a)}{=} P_{e} K r^{-\alpha}+\int_{0}^{2 \pi} \int_{3 R_{c}-r}^{R_{n}-r} \lambda P_{e} K z^{-\alpha} z \mathrm{~d} z \mathrm{~d} \theta \\
& \approx P_{e} K r^{-\alpha}+\frac{2 \pi \lambda P_{e} K}{\alpha-2}\left(3 R_{c}-r\right)^{2-\alpha} .
\end{aligned}
$$

The first term in $(a)$ denotes interference from data cell $o$ while the second term denotes interference from other rings. Consequently, the SIR of the reference UE associated with a BS in the first ring as a result of the inaccurate position estimation can be expressed as

$$
\bar{\gamma}(r)=\frac{\bar{P}_{r}}{\bar{I}_{\text {ext }}}=\frac{(\alpha-2)\left(2 R_{c}-r\right)^{-\alpha}}{(\alpha-2) r^{-\alpha}+2 \pi \lambda\left(3 R_{c}-r\right)^{2-\alpha}} .
$$

Hence, the ASE over the actual coverage of the reference data cell can be expressed as

$$
\begin{gathered}
\mathcal{C}_{e}=\int_{d_{0}}^{R_{c}-l} \ln (1+\widetilde{\gamma}(r)) p_{r}(r) \mathrm{d} r+\int_{R_{c}-l}^{R_{c}} \mathcal{\mathcal { P }}(r, l) \ln (1+\widetilde{\gamma}(r)) p_{r}(r) \mathrm{d} r \\
\quad+\int_{R_{c}-l}^{R_{c}}(1-\mathcal{P}(r, l)) \ln (1+\bar{\gamma}(r)) p_{r}(r) \mathrm{d} r
\end{gathered}
$$

where $\widetilde{\gamma}(r)$ and $\bar{\gamma}(r)$ are defined in (4) and (8), respectively, when errors accumulated as a result of the inaccuracies in the positioning technique is considered. It can be observed that (9) reverts to (5) when $l=0$, i.e., no UE positioning error.

\section{Average Spectral Efficiency: Pathloss And SHADOWING}

\section{A. No error in location information}

For the case where shadowing and pathloss are the dominating factors when modeling the signal propagation, the received signal at the reference UE from the reference BS can be expressed as follows

$$
P_{r, o}=P_{e} K r_{o}^{-\alpha} X_{o},
$$

where $X_{o}$ is a lognormal random variable characterizing the random variation of the received power around the mean value. The PDF of the slowly varying received signal power is given by the standard lognormal expression

$$
p_{X}(s)=\frac{1}{\xi \sigma s \sqrt{2 \pi}} \exp -\left(\frac{\ln (s)-\xi m}{\sqrt{2} \xi \sigma}\right)^{2}
$$

where $\xi=\frac{\ln (10)}{10}, m=\frac{1}{\xi} \ln \left(K P_{e} r_{o}^{-\alpha}\right)$ is the received (logarithmic) mean power in decibels and $\sigma$ is the shadow (logarithmic) standard deviation also in decibels. Similar to (3), the SIR experienced by the reference UE can be expressed as

$$
\tau \approx \frac{P_{e} K r^{-\alpha} X_{o}}{\sum_{j \neq o} P_{e} K r_{j}^{-\alpha} X_{j}}
$$


in an interference limited network. The total interference power is the sum of independent identically distributed (i.i.d.) lognormally distributed random variables. Since the ratio of two lognormal random variables is also a lognormal random variable, the inverse of the $\operatorname{SIR}, \mathcal{F}=\frac{1}{\tau}$, is also lognormally distributed. By using the Fenton-Wilkinson method [17], the mean and variance, $m_{\mathcal{F}}$ and $\sigma_{\mathcal{F}}^{2}$, respectively, of $\mathcal{F}$ can be expressed according to [18] as

$$
\begin{aligned}
m_{\mathcal{F}} & =\frac{1}{\xi} \ln (f(r, \alpha) H(r, \sigma)) \\
\sigma_{\mathcal{F}}^{2} & =2\left(\sigma^{2}-\frac{1}{\xi} \ln (H(r, \sigma))\right)
\end{aligned}
$$

where

$$
\begin{aligned}
H(r, \sigma) & =e^{\frac{\xi^{2} \sigma^{2}}{2}}\left(G(r, \alpha)\left(e^{\xi^{2} \sigma^{2}}-1\right)+1\right)^{-\frac{1}{2}}, \\
G(r, \alpha) & =\frac{\sum_{j \neq o} r_{j}^{-2 \alpha}}{\left(\sum_{j \neq o} r_{j}^{-\alpha}\right)^{2}} \text { and } \\
f(r, \alpha) & \equiv \frac{1}{\widetilde{\gamma}(r, \alpha)}=\frac{\sum_{j \neq o} r_{j}^{-\alpha}}{r^{-\alpha}} .
\end{aligned}
$$

Note that $f(r, \alpha)$ is equivalent to the inverse of the SIR for the case without shadowing, which is given in (4). Hence, $G(r, \alpha)$ can be rewritten as $G(\alpha) \equiv \frac{f(2 \alpha)}{f(\alpha)^{2}}$.

Consequently, the spectral efficiency (SE) of the reference UE positioned at $o$ can be written as

$$
\begin{aligned}
\mathcal{S}(r) & =\int_{0}^{\infty} \ln \left(1+\frac{1}{\mathcal{F}}\right) p_{X}(\mathcal{F}) \mathrm{d} \mathcal{F} \\
& =\frac{1}{\xi \sigma_{\mathcal{F}} \sqrt{2 \pi}} \int_{0}^{\infty} \frac{\ln \left(1+\frac{1}{\mathcal{F}}\right)}{\mathcal{F}} \exp -\left(\frac{\ln (\mathcal{F})-\xi m_{\mathcal{F}}}{\sqrt{2} \xi \sigma_{\mathcal{F}}}\right) \mathrm{d} \mathcal{F},
\end{aligned}
$$

where $p_{X}(\mathcal{F})$ is the PDF of $\mathcal{F}$. Since the expression in $(15)$ is conditioned on the location of the reference UE, it is averaged over the UE's position PDF to obtain the overall ASE over the reference cell as

$$
\begin{aligned}
\overline{\mathcal{C}}_{n}= & \frac{2}{\xi\left(R_{c}-d_{o}\right)^{2} \sqrt{2 \pi}} \int_{d_{0}}^{R_{c}} \frac{\left(r-d_{0}\right)}{\sigma_{\mathcal{F}}} \int_{0}^{\infty} \frac{\ln \left(1+\frac{1}{\mathcal{F}}\right)}{\mathcal{F}} \\
& \exp -\left(\frac{\ln (\mathcal{F})-\xi m_{\mathcal{F}}}{\sqrt{2} \xi \sigma_{\mathcal{F}}}\right)^{2} \mathrm{~d} \mathcal{F} \mathrm{d} r .
\end{aligned}
$$

\section{B. Error in location information}

Here user association as a result of the location error is the same as described for pathloss only scenario in Section III-B. Hence, the received signal by the reference UE as a result of association to the data BS in the first ring can be expressed as

$$
\bar{P}_{r}=P_{e} K\left(2 R_{c}-r\right)^{-\alpha} X_{o}
$$

Since the total interference remains the sum of i.i.d random variables, Fenton-Wilkinson method is used in obtaining the mean and variance of the distribution of $\mathcal{F}$ when the reference $\mathrm{UE}$ is connected to a $\mathrm{BS}$ in the first ring. The mean and variance of the distribution of $\mathcal{F}$ in this case can be obtained from (13), however with the functions $G(r, \alpha)$ and $f(r, \alpha)$ expressed as

$$
\begin{aligned}
G(r, \alpha) & =\frac{r^{-2 \alpha}+\frac{2 \pi \lambda}{2 \alpha-2}\left(3 R_{c}-r\right)^{2-2 \alpha}}{\left(r^{-\alpha}+\frac{2 \pi \lambda}{\alpha-2}\left(3 R_{c}-r\right)^{2-\alpha}\right)^{2}} \text { and } \\
f(r, \alpha) & =\frac{r^{-\alpha}+\frac{2 \pi \lambda}{\alpha-2}\left(3 R_{c}-r\right)^{2-\alpha}}{\left(2 R_{c}-r\right)^{-\alpha}},
\end{aligned}
$$

respectively. Similar to the case without shadowing, two regions can be defined: in the first region, the reference UE is within the range $d_{0}$ to $R_{c}-l$ and errors in its location information do not affect its association and consequently its $\mathrm{SE}$ at that location; whereas in the second region, the reference UE lies in the range $R_{c}-l$ and $R_{c}$ where errors in its location information may affect its association and SE. Consequently, the ASE for the shadowing case with location error can be expressed as

$$
\begin{aligned}
\overline{\mathcal{C}}_{e}= & \frac{2}{\left(R_{c}-d_{0}\right)^{2}}\left(\int_{d_{0}}^{R_{c}-l}\left(r-d_{0}\right) \int_{0}^{\infty} \ln \left(1+\frac{1}{\mathcal{F}}\right) p_{X}(\mathcal{F}) \mathrm{d} \mathcal{F} \mathrm{d} r\right. \\
& +\int_{R_{c}-l}^{R_{c}} \mathcal{P}(r, l)\left(r-d_{0}\right) \int_{0}^{\infty} \ln \left(1+\frac{1}{\mathcal{F}}\right) p_{X}(\mathcal{F}) \mathrm{d} \mathcal{F} \mathrm{d} r \\
& \left.+\int_{R c-l}^{R_{c}}(1-\mathcal{P}(r, l))\left(r-d_{0}\right) \int_{0}^{\infty} \ln \left(1+\frac{1}{\mathcal{F}}\right) \bar{p}_{X}(\mathcal{F}) \mathrm{d} \mathcal{F} \mathrm{d} r\right)
\end{aligned}
$$

where $\bar{p}_{X}(\mathcal{F})$ is the PDF of $\mathcal{F}$ when the reference UE is associated a BS in the first ring. The function $G(r, \alpha)$ and $f(r, \alpha)$ required for obtaining the mean and variance required for $p_{X}(\mathcal{F})$ and $\bar{p}_{X}(\mathcal{F})$ are defined in (14) and (18), respectively. The first term in (19) is a result of the reference UE at position where location errors do no affect its association, while the second and third terms are results of UE at position where location errors may affect its association. It can be observed that (19) revert to (16) when there are no error in association $(l=0)$, i.e., no location error.

\section{Numerical Results And Discussion}

In this section, we present some numerical results to illustrate our analytical findings. Since it has been established in [19] that the SINR of the Poisson stochastic model can be approximated using the fluid model, we model the data BS location according to an homogeneous Poisson Point distribution with density $\lambda$ in our simulation verification. The Poisson modified version of the SINR can be expressed according to the curve fitting approach of [19] as

$$
\gamma_{\phi} \mathrm{dB}=\gamma \mathrm{dB}-\left(a_{0} \alpha-6\right),
$$

where the parameter $a_{0}$ depends on the shadowing standard deviation $\sigma$ and the error radius $l$. For the case with $l=0$ and $l>10 \mathrm{~m}$, the parameter $a_{0}=\{1.165,1.175,1.22,1.39\}$ and $\{1.22,1.23,1.28,1.47\}$, respectively, for $\sigma=\{0,2,4,6\} \mathrm{dB}$. The data cell density is related to the average data cell radius such that $\lambda=\frac{1}{\pi R_{c}^{2}}$, hence $R_{c}$ can be set to fix $\lambda$. The number of data $\mathrm{BS}$ is obtained according to a Poisson distribution of parameter $\lambda \mathcal{A}$, where $\mathcal{A}$ is the area of the ROI which is chosen 


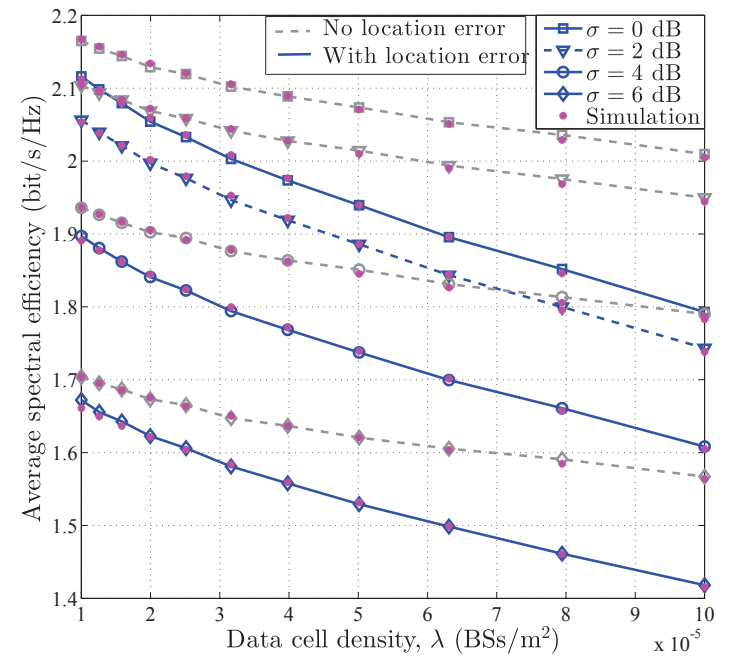

Fig. 3. Impact of data cell density on ASE for $\alpha=3.25, l=25$.

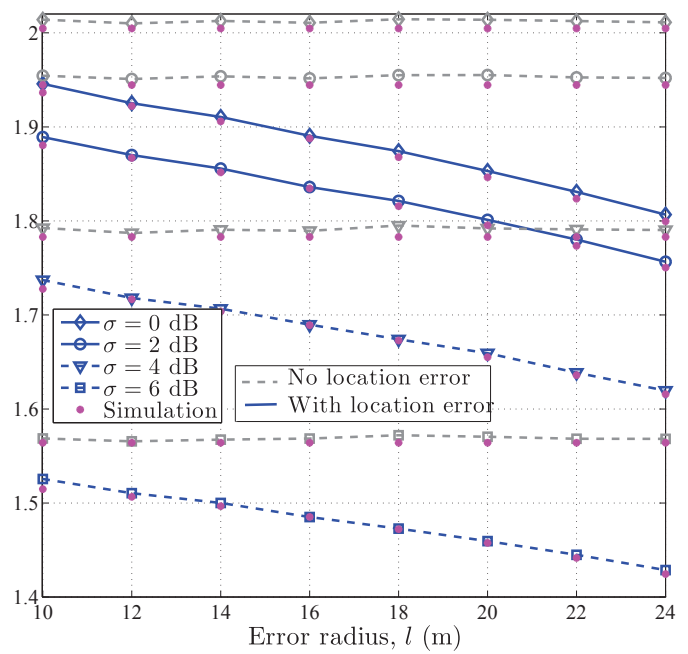

Fig. 4. Impact of error radius on ASE for $\alpha=3.25, \lambda=$ $10^{-4} \mathrm{BS} / \mathrm{m}^{2}$.

to obtain an average of 200 data BS in the area. The SINR and SE of a typical UE at a random location $\left(x_{i}, y_{i}\right)$ can then be obtained. Subsequently, the ASE over the entire area is obtained.

In case of location error, the reported location is obtained by generating pseudo random, pseudo independent numbers $u_{i}$ and $v_{i}$ uniformly distributed in $[-1,1]$ and $[0,1]$, respectively, such that the reported coordinates is $\left(x_{i}+l u_{i} \cos \left(2 \pi v_{i}\right), y_{i}+\right.$ $\left.l u_{i} \sin \left(2 \pi v_{i}\right)\right)$. The UE-data BS association and the corresponding SE is evaluated based on the reported position. Subsequently, the ASE over the area is obtained.

In Figs. 3 and 4, we plot the ASE against the data cell density $\lambda$ and circular error radius $l$, respectively, for scenarios

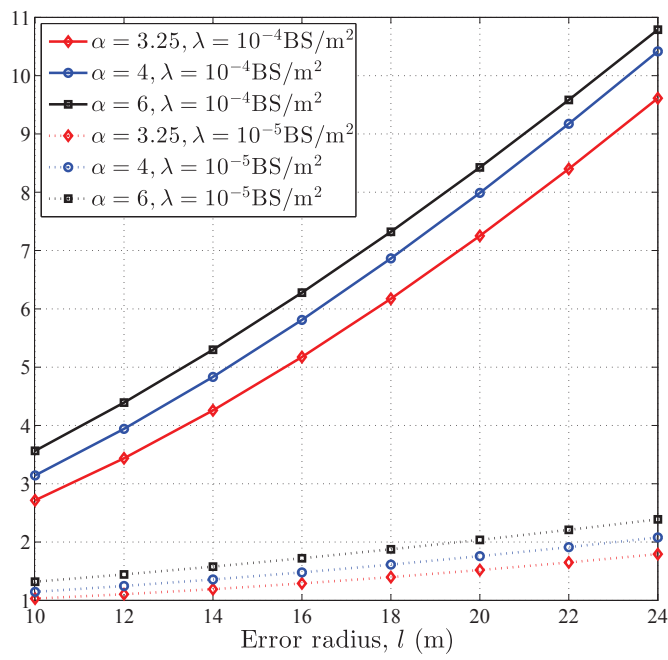

Fig. 5. Degradation in average spectral efficiency as a function of the pathloss exponent and DBS density (no shadowing).

with and without location error, and for standard deviation, $\sigma=0,2,4,6 \mathrm{~dB}$. The results in both figures shows a close match between the analytical and simulation results. Fig. 3 shows that errors in estimating the position of the UEs leads to degradation in the ASE, which is more severe in very dense data BS deployment such as $\lambda=10^{-4} \mathrm{BS} / \mathrm{m}^{2}$. This is due to the fact that the tendency of a typical UE associating with another BS other than the closest data BS increases as the data BS density increases. Fig. 4 shows that for a fixed data BS density, the ASE decreases as the error radius increases. This is because the likelihood of associating with a less optimal data $\mathrm{BS}$ increases as the error radius increases.

In Fig. 5, we plot the degradation in the ASE as a result of the errors in the UE location information for pathloss exponents, $\alpha=3.25,4$ and 6 , DBS densities, $\lambda=10^{-4}$ and $10^{-5} \mathrm{BS} / \mathrm{m}^{2}$. Similar to Fig. 4 , the results show that the performance of the database-aided scheme is highly dependent on the accuracy of the positioning technique since the ASE degrades significantly as the UE location error radius increases. Furthermore, the degradation in performance is more severe at high pathloss exponent. This is because wrong association of the UE at high pathloss exponent leads to a more pronounced reduction in the received signal strength.

A key finding from our results is that the gains attributed to database-aided networks [11] can only be achieved by having very accurate positioning estimation techniques, which is very difficult to achieve in an outdoor environment. Hence, the degradation effect from inaccurate position estimation must be taken into consideration when defining the system specifications, such as peak data rate and area spectral efficiency, for database-aided networks. 


\section{CONCLUSION}

In this paper, we have investigated the impact of using database-aided user association in the control-data separated architecture. Based on the fluid model interference analysis technique, we have analyzed the average spectral efficiency (ASE) in the data-plane of the control-data separated architecture. The numerical results suggest that the ASE degrades as the accuracy of the user positioning technique decreases. Furthermore, this degradation can be very severe when the data BS density is very high.

In future work, it would be interesting to analyze the impact of the accuracy of the positioning technique on the achievable energy savings in database-aided networks.

\section{ACKNOWLEDGMENT}

This work was made possible by NPRP grant No. 5-10472437 from the Qatar National Research Fund (a member of The Qatar Foundation). The statements made herein are solely the responsibility of the authors.

We would like to acknowledge the support of the University of Surrey 5GIC members for this work.

\section{REFERENCES}

[1] J. Andrews, S. Buzzi, W. Choi, S. Hanly, A. Lozano, A. Soong, and J. Zhang, "What Will 5G Be?" IEEE J. Sel. Areas Commun., vol. 32, no. 6, pp. 1065-1082, Jun. 2014.

[2] A. Imran, A. Zoha, and A. Abu-Dayya, "Challenges in 5G: How to Empower SON with Big Data for Enabling 5G," IEEE Netw., vol. 28, no. 6, pp. 27-33, Nov. 2014.

[3] D. Lopez-Perez, I. Guvenc, G. de la Roche, M. Kountouris, T. Quek, and J. Zhang, "Enhanced Intercell Interference Coordination Challenges in Heterogeneous Networks," IEEE Wireless Commun. Mag., vol. 18, no. 3, pp. 22-30, Jun. 2011.

[4] H. Ishii, Y. Kishiyama, and H. Takahashi, "A Novel Architecture for LTE-B :C-plane/U-plane Split and Phantom Cell Concept," in Proc. IEEE Globecom Workshops (GC Wkshps), Dec. 2012, pp. 624-630.

[5] A. Zakrzewska, D. López-Pérez, S. Kucera, and H. Claussen, "Dual Connectivity in LTE HetNets with Split Control- and User-Plane," in Proc. IEEE Globecom Workshops, Dec. 2013, pp. 391-396.
[6] A. Mohamed, O. Onireti, Y. Qi, A. Imran, M. Imran, and R. Tafazolli, "Physical Layer Frame in Signalling-Data Separation Architecture: Overhead and Performance Evaluation," in Proc. European Wireless Conference, May 2014.

[7] A. Mohamed, O. Onireti, M. A. Imran, A. Imran, and R. Tafazolli, "Correlation-based Adaptive Pilot Pattern in Control/Data Separation Architecture," in Proc. IEEE International Conference on Communications (ICC), 2015.

[8] _ _ "Control-Data Separation Architecture for Cellular Radio Access Networks: A Survey and Outlook," IEEE Commun. Surveys Tuts., Jun. 2015.

[9] 3rd Generation Partnership Project (3GPP), Technical Specification Group Radio Access Network "Views on Small Cell On/Off Mechanisms, 3GPP TSG RAN, 3GPP Std. R1-133 456," Aug. 2013.

[10] _ "Enhancement of Small Cell On/Off, 3GPP TSG RAN 3GPP Std. R1-132 888," Aug. 2013.

[11] E. Ternon, P. Agyapong, L. Hu, and A. Dekorsy, "Database-aided Energy Savings in Next Generation Dual Connectivity Heterogeneous Networks," in Proc. IEEE WCNC, Apr. 2014, pp. 2811-2816.

[12] —_ "Energy Savings in Heterogeneous Networks with Clustered Small Cell Deployments," in Proc. ISWCS, Aug. 2014, pp. 126-130.

[13] T. Kos, M. Grgic, and G. Sisul, "Mobile User Positioning in GSM/UMTS Cellular Networks," in Proc. 48th International Symposium ELMAR, Jun. 2006, pp. 185-188.

[14] J.-M. Kelif, M. Coupechoux, and G. Philippe, "A Fluid Model for Performance Analysis in Cellular Networks," EURASIP Journal onWireless Communications and Networking, Jun. 2010.

[15] I. Akbari, O. Onireti, M. A. Imran, A. Imran, and R. Tafazolli, "Effect of Inaccurate Position Estimation on Self-Organising Coverage Estimation in Cellular Networks," in European Wireless Conference, May 2014.

[16] M.-S. Alouini and A. Goldsmith, "Area Spectral Efficiency of Cellular Mobile Radio Systems," IEEE Trans. Veh. Technol., vol. 48, no. 4, pp. 1047-1066, Jul. 1999.

[17] L. Fenton, "The Sum of Log-Normal Probability Distributions in Scatter Transmission Systems," IRE Transactions on Communications Systems, vol. 8, no. 1, pp. 57-67, Mar. 1960.

[18] J.-M. Kelif and M. Coupechoux, "Impact of Topology and Shadowing on the Outage Probability of Cellular Networks," in Proc. IEEE International Conference on Communications, Jun. 2009.

[19] J.-M. Kelif, S. Senecal, M. Coupechoux, and C. Bridon, "Analytical Performance Model for Poisson Wireless Networks with Pathloss and Shadowing Propagation," in Proc. IEEE Globecom Workshops (GC Wkshps), 2014, Dec. 2014, pp. 1528-1532. 